\title{
Application of Bond Constraint Theory to the Switchable Optical Memory Material $\mathrm{Ge}_{2} \mathrm{Sb}_{2} \mathrm{Te}_{5}$
}

\author{
D. A. Baker, ${ }^{1}$ M. A. Paesler, ${ }^{1}$ G. Lucovsky, ${ }^{1}$ S. C. Agarwal, ${ }^{1, *}$ and P. C. Taylor ${ }^{2}$ \\ ${ }^{1}$ Physics Department, North Carolina State University, Raleigh, North Carolina 27695-8202, USA \\ ${ }^{2}$ Physics Department, Colorado School of Mines, Golden, Colorado 80401-1887, USA
}

(Received 24 March 2006; published 28 June 2006)

\begin{abstract}
A new extended x-ray-absorption fine structure spectroscopy study of local bonding identifies for the first time significant concentrations of Ge-Ge bonds in amorphous $\mathrm{Ge}_{2} \mathrm{Sb}_{2} \mathrm{Te}_{5}$. The study provides a new understanding of the local molecular structure of this phase-change material. Application of bond constraint theory indicates that the amorphous phase is an ideal network structure in which the average number of constraints per atom equals the network dimensionality. Analysis within this framework imparts new and significant insights concerning the nature of the reversible optically driven amorphouscrystalline phase transition of $\mathrm{Ge}_{2} \mathrm{Sb}_{2} \mathrm{Te}_{5}$.
\end{abstract}

DOI: 10.1103/PhysRevLett.96.255501

PACS numbers: 61.43.Bn, 61.43.Fs, 78.70.Dm

Switching in chalcogenide glasses has attracted a good deal of attention [1]. Present interest in these glasses is driven by the ability of a particular composition $\left(\mathrm{Ge}_{2} \mathrm{Sb}_{2} \mathrm{Te}_{5}\right.$, or GST) to be repeatedly switched between crystalline $(c)$ and amorphous $(a)$ states by application of light or electrical pulses of suitable intensities and durations [2]. Differences in the properties of the $a$ and $c$ materials (e.g., reflectivity) have allowed for device applications $[3,4]$. Knowledge of the nature of the $a-c$ transition is crucial to understanding the switching operation of GST.

The present study uses bond constraint theory [5-7] to afford, for the first time, new insights about the reversibility of the $a-c$ transition in GST. Bond constraint theory provides a framework for understanding $a$ materials much as periodicity does for $c$ materials. In relating the average bonding constraints of an $a$ network to the dimensionality of the network, the theory allows for the understanding of an $a$ material's stability.

To exploit the strengths of bond constraint theory in a study of the switching behavior of GST, one needs a tool to investigate the bonding characteristics of the $a$ material. Extended x-ray absorption fine structure, or EXAFS, provides just such a tool [8]. EXAFS involves a careful analysis of the oscillating part of the absorption coefficient of a material at energies above the absorption edge. This leads to information relating to the environment of a central excited atom. Based on EXAFS data - and contrary to the other interpretations of such results [3] - this work unambiguously identifies the important role of $\mathrm{Ge}-\mathrm{Ge}$ bonds and their profound effect on the properties of GST.

$\mathrm{Ge}_{2} \mathrm{Sb}_{2} \mathrm{Te}_{5}$ films were $\mathrm{rf}$ sputtered onto aluminum foil substrates to a thickness of $2.7 \mu \mathrm{m}$. The samples were sliced into $10 \mathrm{~mm} \times 4 \mathrm{~mm}$ sections and stacked, creating 8 layers with an effective film thickness of $\sim 22 \mu \mathrm{m}$. We measured the $K_{1^{\prime}}$ edges of $\mathrm{Ge}, \mathrm{Sb}$, and Te in transmission mode using a $\mathrm{Si}(111)$ double-crystal monochromator at line ID-10 (MRCAT) of the Advanced Photon Source at Argonne National Laboratory. Incident and transmitted photons were measured at room temperature with ioniza- tion chambers filled with $\mathrm{Ar}+15 \% \mathrm{Kr}$ and $\mathrm{Kr}$, respectively. The measured edges are shown in Fig. 1. The $k^{3}$-weighted EXAFS spectra for all three edges are displayed as the solid line in Fig. 2. The transformed spectra were fit using the Athena and Artemis analysis packages [9], which utilize the FEFF [10] and IFEFFIT [11] codes. A small cluster molecular model was used as a way to avoid any bias toward crystallinity in the analysis. All three possible backscattering species were used, and evidence of the lack of a scattering pair was manifest in either nonphysical parameters and/or an unacceptably large (typically $>700$ ) reduced chi-squared error obtained between the FEFF modeled pairs and observed data. Of the six possible atom pairs, only three showed fits with acceptable

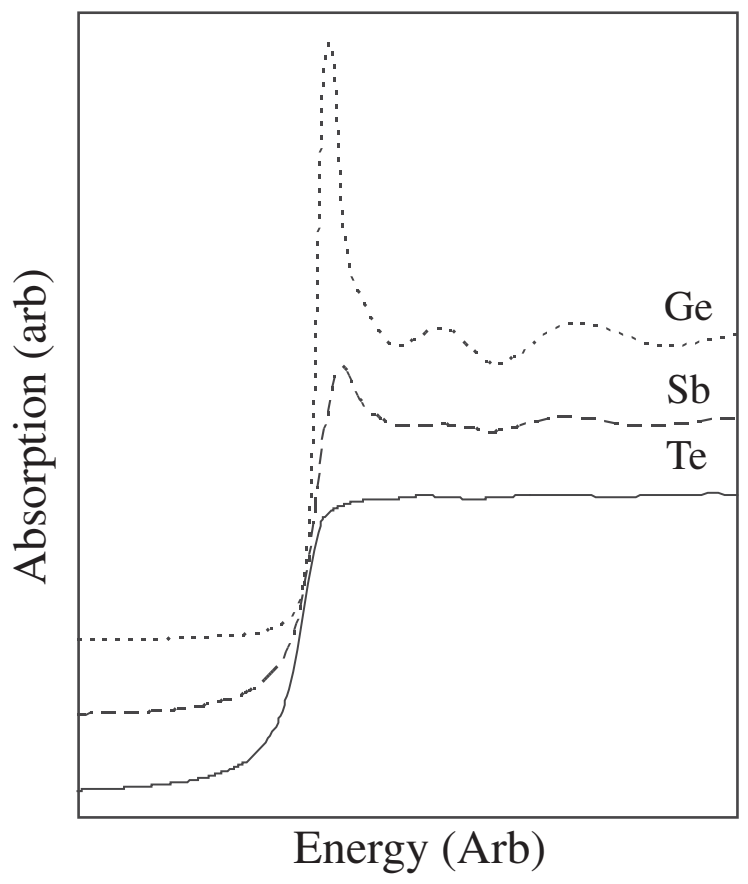

FIG. 1. Normalized absorption spectra for all three atomic species, shifted in energy for comparison. 


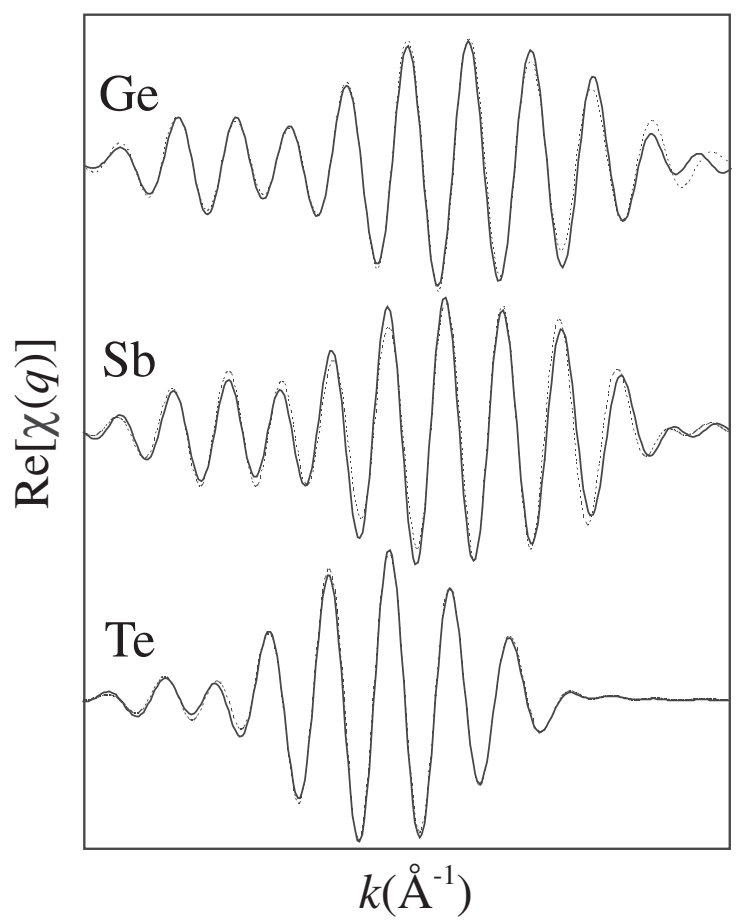

FIG. 2. Plots of $k^{3}$-weighted normalized EXAFS spectra for $\mathrm{Ge}_{2} \mathrm{Sb}_{2} \mathrm{Te}_{5}$. Solid lines are data; dashed lines are fits.

chi-squared errors. Attempts to fit data with Te-Te, Sb-Sb, and $\mathrm{Ge}-\mathrm{Sb}$ bonds failed to meet the criteria of acceptable fits (physically reasonable parameters and reduced chisquared error less than around 600) and were eliminated as possible bonding pairs. In particular, the $\mathrm{Ge}-\mathrm{Sb}$ pair could not be fit using either Ge data or Sb data.

Table I presents a summary of the coordination $(N)$ and nearest neighbor distances for the four atom pairs. Consistency in the results is clear as the Te nearest neighbor distances $\left(R_{\mathrm{Te}-\mathrm{Sb}}=2.83 \AA\right.$ and $\left.R_{\mathrm{Te}-\mathrm{Ge}}=2.62 \AA\right)$ are found to be the same, within the error, regardless of whether they were determined from Te-edge data or from the neighboring atom data ( $\mathrm{Sb}$ and $\mathrm{Ge}$, respectively). Also note that the reported bond lengths agree with tabulated covalent radii for each species [12]. $N$ determinations indicate fully coordinated $\mathrm{Ge}$ and $\mathrm{Sb}$ with $N_{\mathrm{Ge}}=3.9 \pm$

TABLE I. Coordination numbers and nearest neighbor bond distances for $a-\mathrm{Ge}_{2} \mathrm{Sb}_{2} \mathrm{Te}_{5}$ as determined by EXAFS. Internal consistency is manifest in identical values (within the error) for heteropolar bonding distances when using x-ray absorption data from either atomic species.

\begin{tabular}{cccc}
\hline \hline Atom & Bond & Coordination & $R(\AA)$ \\
\hline $\mathrm{Ge}$ & $\mathrm{Ge}-\mathrm{Te}$ & $3.3 \pm 0.5$ & $2.63 \pm 0.01$ \\
& $\mathrm{Ge}-\mathrm{Ge}$ & $0.6 \pm 0.2$ & $2.47 \pm 0.03$ \\
$\mathrm{Sb}$ & $\mathrm{Sb}-\mathrm{Te}$ & $2.8 \pm 0.5$ & $2.83 \pm 0.01$ \\
$\mathrm{Te}$ & $\mathrm{Te}-\mathrm{Ge}$ & $1.2 \pm 0.3$ & $2.62 \pm 0.01$ \\
& $\mathrm{Te}-\mathrm{Sb}$ & $1.2 \pm 0.3$ & $2.83 \pm 0.01$ \\
\hline \hline
\end{tabular}

0.8 and $N_{\mathrm{Sb}}=2.8 \pm 0.5$ and slightly overcoordinated Te with $N_{\mathrm{Te}}=2.4 \pm 0.6$.

Similarities in photoelectron backscattering of $\mathrm{Sb}$ and $\mathrm{Te}$ render it quite difficult, if not impossible, to differentiate between $\mathrm{Sb}-\mathrm{Sb}$ bonds and $\mathrm{Sb}-\mathrm{Te}$ bonds in the EXAFS data. Chemical preference, however, provides us with a guide to the nature of the bonding environment, and allows for a general argument in favor of one bond type over another. Bond energies for Sb-Te (40.6 kpm) and Sb-Sb (39.6 kpm) [13] support the assumption that the Sb-Te bonds account for most, if not all, of the backscattering signal, which is reflected in our results.

Assuming the only like-atom bonds are Ge-Ge bonds, and $N_{\mathrm{av}}(\mathrm{Te})=2.4$, then $1 / 7(\sim 14 \%)$ of the Ge bonds are homopolar. This agrees, within the error, with the results shown above for Ge-Ge bonding. Based on these EXAFS results, the presence of homopolar bonding of $\mathrm{Ge}$ suggests a model where all $\mathrm{Ge}$ atoms are bonded to one $\mathrm{Ge}$ atom and three Te atoms in $\mathrm{G}_{2} \mathrm{Se}_{3}$ type local bonding arrangement [14]. Sb atoms are then interspersed evenly throughout the structure with three Te neighbors in $\mathrm{Sb}_{2} \mathrm{Te}_{3}$ arrangements.

Bond constraint theory $[5,6]$ demonstrates that in order for an alloy to be a good glass former, the average number of constraints per atom, $C_{\mathrm{av}}$, must be approximately equal to the dimensionality $D$ of the network, that is $C_{\mathrm{av}} \approx D$. In the present case $D=3$. For the present system if $C_{\mathrm{av}}$ is significantly greater or less than 3 , enthalpy and entropy effects, respectively, tend to make stable, low-defect glass formation difficult. When $C_{\mathrm{av}} \approx D$, however, neither the entropy effects nor the long-range order enthalpy effects dominate, and a stress-free rigid network is formed. This is a small compositional region in which the increase of rigidity accompanies an increase in $C_{\mathrm{av}}$, and the composition makes the transition from under-constrained, or floppy, to over-constrained, or stressed-rigid material. The onset of regions of rigidity begins just below and rigidity percolation occurs just above this critical number of average constraints. The EXAFS data coupled with constraint theory allow for determination of $C_{\mathrm{av}}$ for the system, and this is accomplished by considering each atomic species in turn.

Consider first Ge. Given that homopolar bonds exist in the alloy, one can calculate the average constraints for the system. To zeroth order, a tetrahedral Ge configuration yields a $C_{\text {av }}$ of 7 . This is, however, too simplified, as bond-bending constraints can be removed by considering in detail the specific bonding arrangements, in this instance $\mathrm{Ge}_{2} \mathrm{Te}_{3}$. In particular, considering bending constraints around the Ge atom, the force constant for the Ge-Ge-Te bending motion is significantly reduced with respect to that of a Te-Ge-Te bending motion due to the different $\mathrm{Ge}-\mathrm{Ge}-$ $\mathrm{Te}$ and $\mathrm{Te}-\mathrm{Ge}-\mathrm{Te}$ bond energies. This permits the removal of 2.67 bending constraints for the $\mathrm{Ge}_{2} \mathrm{Te}_{3}$ arrangement. Our EXAFS results suggest a bonding model in which all Ge atoms are in this arrangement, thus the total number of 
constraints around the average $\mathrm{Ge}$ atom is reduced from 7 ( 5 bending) to 4.33 ( 2.33 bending).

Consider next $\mathrm{Sb}$. There are 1.5 stretching constraints and 3 bending constraints, resulting in 4.5 total constraints. None of these constraints are broken; therefore, one finds the total constraints around the $\mathrm{Sb}$ atoms to be 4.5.

Finally, consider Te. Table I shows that $\mathrm{Te}$ is over coordinated. A simple bond-counting exercise supports this result. The proposed model gives $\mathrm{Ge}_{2} \mathrm{Sb}_{2} \mathrm{Te}_{5}$ as a combination of $\mathrm{Ge}_{2} \mathrm{Te}_{3}$ and $\mathrm{Sb}_{2} \mathrm{Te}_{3}$ structural units. This counting results in a deficiency in Te for the GST composition, as stoichiometry requires that the addition of these two units equal $\mathrm{Ge}_{2} \mathrm{Sb}_{2} \mathrm{Te}_{6}$. This $1 / 6$ or $\sim 17 \% \mathrm{Te}$ deficiency is reflected in our results, as the percent of overcoordinated Te determined from these fits is $0.4 / 2.4 \sim$ $17 \%$. A Te deficiency, combined with $8-N$ coordination of $\mathrm{Ge}$ and $\mathrm{Sb}$, requires that some Te atoms over coordinate, resulting in the presence of both twofold and planarthreefold geometries. No constraints can be removed for the former configuration, and in the latter, the bond order is reduced from one electron/bond to $2 / 3$ electrons/bond. Constraints are removed for this configuration, but proportionately so [7], resulting in 2 constraints for planarthreefold coordinated $\mathrm{Te}$, as well as twofold coordinated $\mathrm{Te}$. Therefore, the number of total constraints for all $\mathrm{Te}$ atoms is 2 .

The total number of constraints for the entire $\mathrm{Ge}_{2} \mathrm{Sb}_{2} \mathrm{Te}_{5}$ alloy follows: Ge contribution: $4.33 \times 2=8.66$; Sb contribution: $4.5 \times 2=9$; Te contribution: $2 \times 5=10$. Thus,

$$
C_{\mathrm{av}}=\frac{8.66+9+10}{9}=3.07 \text {. }
$$

This value of $C_{\mathrm{av}}$ nears the ideal value of 3 suggested for a material in the stress-free state, and more importantly, for a good glass former.

The argument presented above, based on constraint theory and EXAFS results, suggests that GST lies in a region of intermediate phase within the ternary composition diagram. It is in this region where entropy effects in noncrystalline phase and enthalpy effects in the crystalline phase combine to make a reversible amorphous to crystalline phase transitions favorable. Future work, therefore, must delve more deeply into alloys close to GST in the $\mathrm{Ge}-\mathrm{Sb}$-Te system in order to identify and quantify the microscopic origin of changes in the amorphous to crystalline transitions, e.g., the sharpness of the transition, and the change in transmissivity as discussed by Yamada et al. [4]. EXAFS, vibrational spectroscopies, bond constraint theory, and other approaches provide a pathway for obtaining a deeper understanding of the behavior of this family of materials, and the local bonding and resultant electronic structure.

We thank A. Edwards H. Fritzsche, S. J. Hudgens, S. R. Ovshinsky, and J. C. Phillips for fruitful discussions. This work was begun under the direction of our colleague, the late Professor Dale Sayers, whose many contributions to the development of EXAFS and the study of amorphous materials provide a legacy to our field and ongoing benefit to us all. Work supported by the Air Force Research laboratory under Grant No. F29601-03-01-0229 and by the National Science Foundation under Grant No. DMR 0307594. Use of the Advanced Photon Source was supported by the U. S. Department of Energy, Office of Science, Office of Basic energy Sciences, under Contract No. W-31-109-ENG-38. MRCAT operations are supported by the Department of Energy and the MRCAT member institutions .

*Currently at Department of Physics, Indian Institute of Technology, Kanpur 208 016, India.

[1] S. R. Ovshinsky, Phys. Rev. Lett. 21, 1450 (1968).

[2] J. K. Olson, H. Li, and P.C. Taylor, Journal of Ovonic Research 1, 1 (2005).

[3] A. V. Kolobov, P. Fons, A. I. Frenkel, A. L. Ankudinov, J. Tominaga, and T. Uruga, Nature Mater. 3, 703 (2004).

[4] N. Yamada, E. Ohno, K. Nishiuchi, and N. Akahira, J. Appl. Phys. 69, 2849 (1991).

[5] J. C. Phillips, J. Non-Cryst. Solids 34, 153 (1979).

[6] M. F. Thorpe, J. Non-Cryst. Solids 57, 355 (1983).

[7] R. Kerner and J. C. Phillips, Solid State Commun. 117, 47 (2000).

[8] D. E. Sayers, F. W. Lyttle, and E. A. Stern, Phys. Rev. B 11, 4836 (1975).

[9] B. Ravel, J. Synchrotron Rad. 12, 537 (2005).

[10] J. J. Rehr, J. Mustre de Leon, S.I. Zabinsky, and T. C. Albers, J. Am. Chem. Soc. 113, 5135 (1991).

[11] M. Newville, J. Synchrotron Rad. 8, 322 (2001).

[12] F. A. Cotton and G. Wilkinson, Advanced Inorganic Chemistry (Interscience Publishers, New York, 1972), 3rd ed., Chap. 3, p. 117, Table 3.4.

[13] R. T. Sanderson, Chemical Bonds and Bond Energy (Academic, New York, 1976), 2nd ed., Chap. 3, pp. 6063.

[14] A. Feltz, Amorphe und Glasartige Anorganische Festkörper (Akademi-Verlag, Berlin, 1983), p. 248. 